\title{
Sex-Specific Plasticity Explains Genetic Variation in Sexual Size Dimorphism in Drosophila
}

\author{
Isabelle M Vea ${ }^{1}$, Austin Wilcox ${ }^{1}$, W. Anthony Frankino ${ }^{2}$, Alexander W Shingleton ${ }^{1 *}$ \\ ${ }^{1}$ Department of Biological Sciences, University of Illinois at Chicago, Chicago, IL 60607, USA \\ ${ }^{2}$ Department of Biology and Biochemistry, University of Houston, Houston, TX 77204, USA \\ *Email: ashingle@uic.edu
}

Keywords: sexual dimorphism, body size, development, plasticity, nutrition

\begin{abstract}
The difference in body size between females and males, or sexual size dimorphism (SSD), is almost ubiquitous, and yet we have a remarkably poor understanding of the developmental-genetic mechanisms that generate it. Such an understanding is important if we are to distinguish between the many theoretical models of SSD evolution. One such model is the condition dependence hypothesis, which proposes that the body size of the larger sex is also more environmentally sensitive, a phenomenon called sex-specific plasticity (SSP). Because SSP generates differences in female and male body size, selection on plasticity may underlie the evolution of sexual size dimorphism. To test this hypothesis, however, we need to know the genetic architecture of both SSD and SSP, which is challenging because both are characteristics of populations not individuals. Here, we overcome this challenge by using isogenic lineages of Drosophila to measure both SSD and SSP for a genotype. We demonstrate extensive genetic variation for SSD among genotypes that is tightly correlated with variation in SSP, indicating that the same developmental-genetic mechanisms
\end{abstract}


regulate both phenomena. These data support the condition dependence hypothesis and suggest that the observed SSD is a consequence of selection on the developmental-genetic mechanisms that regulate SSP.

\section{Introduction}

Sexual Size Dimorphism (SSD), the difference in body size between males and females, is perhaps the most familiar and widespread form of sexual dimorphism. SSD is extremely variable among species regardless of their average size, and varies in both direction and magnitude [1,2]. For example, most mammals and birds have male-biased SSD, where males are larger than females $[3,4]$, whereas insects typically exhibit female-biased SSD [5]. SSD is also highly evolutionarily labile, even among closely related species and populations within species [6-9] suggesting that there is considerable intraspecific genetic variation in SSD upon which selection can act. While numerous hypotheses have been proposed regarding the selective pressures that might give rise to SSD [10], the developmental-genetic mechanisms that are the target of these selective agents remain largely unknown. This represents a substantial gap in our understanding of the evolution of SSD, since knowing the targets of selection could provide insight regarding the modes of selection favoring sexspecific changes in body size. Hampering efforts to fill this gap is the challenge of describing genetic variation in SSD within a population, since SSD cannot be measured for an individual.

For SSD to evolve, the strength or direction of selection on male and female body size must necessarily differ. For example, sex-specific selection on body size can produce male-biased SSD when focused on display or fighting ability, whereas female-biased SSD can result from fecundity selection, which also typically correlates with body size. Regardless of the ultimate selective pressures that generate SSD, theory suggests that the larger sex gains greater fitness benefits from increased body size than does the smaller sex. A consequence of this is that we might expect the larger sex to show a greater response to environmental factors that enhance body size. This 
heightened sensitivity is referred to as the condition dependence hypothesis. Condition dependence is a form of phenotypic plasticity whereby the expression of a trait changes with the phenotypic condition of the individual [11]. The condition dependence hypothesis was originally developed to explain the evolution of sexually-selected traits that signal mate quality in a reliable, conditiondependent manner [11-15]. However, the condition dependent hypothesis can be applied more generally to any trait that is differentially selected in males and females, regardless of the mode of selection [6]. If individuals in good condition enjoy larger marginal benefits from increasing trait size in one sex relative to the other, this sex should show greater investment in the trait, be it overall body size or the size of an individual organ $[16,17]$. In as much as condition reflects environmental variation, particularly access to nutrition during growth, the condition dependent hypothesis predicts a positive correlation between SSD and size plasticity such that the larger sex will exhibit greater size plasticity than the smaller sex [18]. From a quantitative genetic perspective, this sexspecific plasticity (SSP) can be considered a $G \times E$ interaction, where $G$ is sex and $E$ is environmental variation.

Several studies provide evidence for a correlation between sexual dimorphism and sex-specific plasticity, either by comparing multiple dimorphic and non-dimorphic traits within species, or by comparing the same trait across multiple species. For example, the strength of condition dependence increases with the degree of sexual dimorphism among different traits in Prochyliza xanthostoma and Telostylinus angusticollis flies [12,19]: traits that are more dimorphic show greater differential plasticity between sexes. The same pattern is observed for sexually-dimorphic traits among species [20]. For the specific example of SSD, where the trait is body size as a whole, comparative studies among species also support the condition dependent hypothesis. In arthropods, where SSD is female-biased, there is a general trend for female body size to be more plastic[18,21]. Among birds and mammals, where SSD is typically male-biased, the opposite is true [22,23]. Further, among the few arthropod taxa with male-biased SSD, males body size also appears to be more plastic than female body size [6]. 
What is unclear from these studies, however, is the origin of these correlations. One possibility is that SSP evolves as a consequence of SSD, with selection targeting different developmental-genetic mechanisms for each. Indeed, early theoretical models of the condition dependent hypothesis argue that sexually dimorphic traits are more likely to evolve if the loci that regulate expression of the traits are distinct from but linked to loci that cause the traits to be condition-dependent [11]. Alternatively, selection for SSD may target the same developmental-genetic mechanisms that increase size under better conditions, that is, selection may focus on the mechanisms that regulate size plasticity. From this perspective, SSD evolves as a consequence of sex-specific responses to changes in condition. Distinguishing between these hypotheses requires insight into whether SSD and SSP covary among individual genotypes within a population, which is not provided by existing data.

Here we investigate whether SSD and SSP are genetically correlated among individuals within a population by manipulating nutrition and measuring body size and body-size plasticity in 196 isogenic $D$. melanogaster lineages. We found considerable genetic variation for SSD among genotypes, and determined that variation in SSD and SSP are genetically correlated. These data support the hypothesis that same loci regulate both SSD and SSP and suggest that the observed SSD is a consequence of selection for SSP.

\section{Results}

\section{Sexual Size Dimorphism shows genetic variation in Drosophila melanogaster}

We found significant genetic variation in SSD among the 186 DGRP lineages that we measured (Fig. 1), using pupal area of fed flies as a measure of overall body size [24]. Specifically, a linear mixed effect model that included random variation in SSD among lineages was a significantly better fit to the data than a model that did not (Table 1). Further, SSD is female biased (Best Linear Unbiased Predictions: male body size $=14.47$, female body size $=14.56$ ) . 

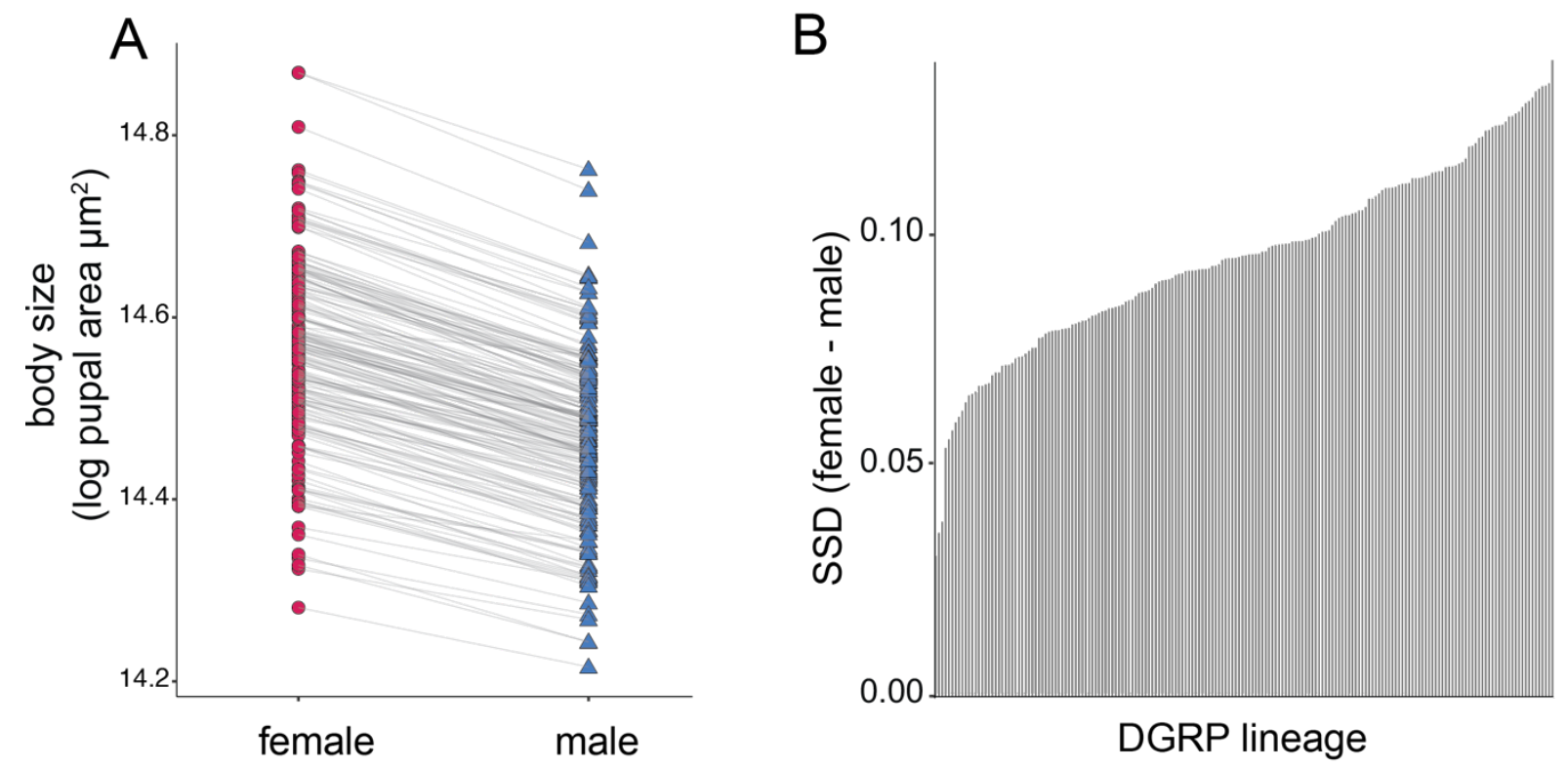

Figure 1: Variation in Sexual Size Dimorphism (SSD) among isogenic lineages. (A) Female and male body size for each of the 186 lineages. Estimates of body size are Best Linear Unbiased Predictions (BLUPS) from a mixed model linear regression (Table 1: Model 1). Sexes for each lineage are connected by a line. (B) Ranked plot of SSD for these lineages (log body size female $-\log$ body $_{\text {size }}$ male).

\section{Variation in SSD reflects variation in female body size more than male body size}

To determine the degree to which SSD results from variation in male versus female size, we fit linear relationships between SSD $(y)$ and either male or female body size (x) (Fig. 2A). If variation in SSD is driven by variation in female size more than variation in male size, the slope of this relationship should be steeper for females than for males, and the $R^{2}$ should be higher. We found that the slope of the relationship between body size and SSD was significantly steeper for females than males (t-test on the slope, $P<2 \mathrm{e}-16)$, and $R^{2}$ was also significantly higher for female size than male size against SSD $\left(\mathrm{R}^{2}(\right.$ female $)=0.29,95 \% \mathrm{Cl}: 0.18-0.39 ; \mathrm{R}^{2}$ (male $\left.)=0.14,95 \% \mathrm{Cl}: 0.05-0.23\right)$, Fig. 2A). These data indicate that variation in SSD among lineages was due primarily to variation in female size. 

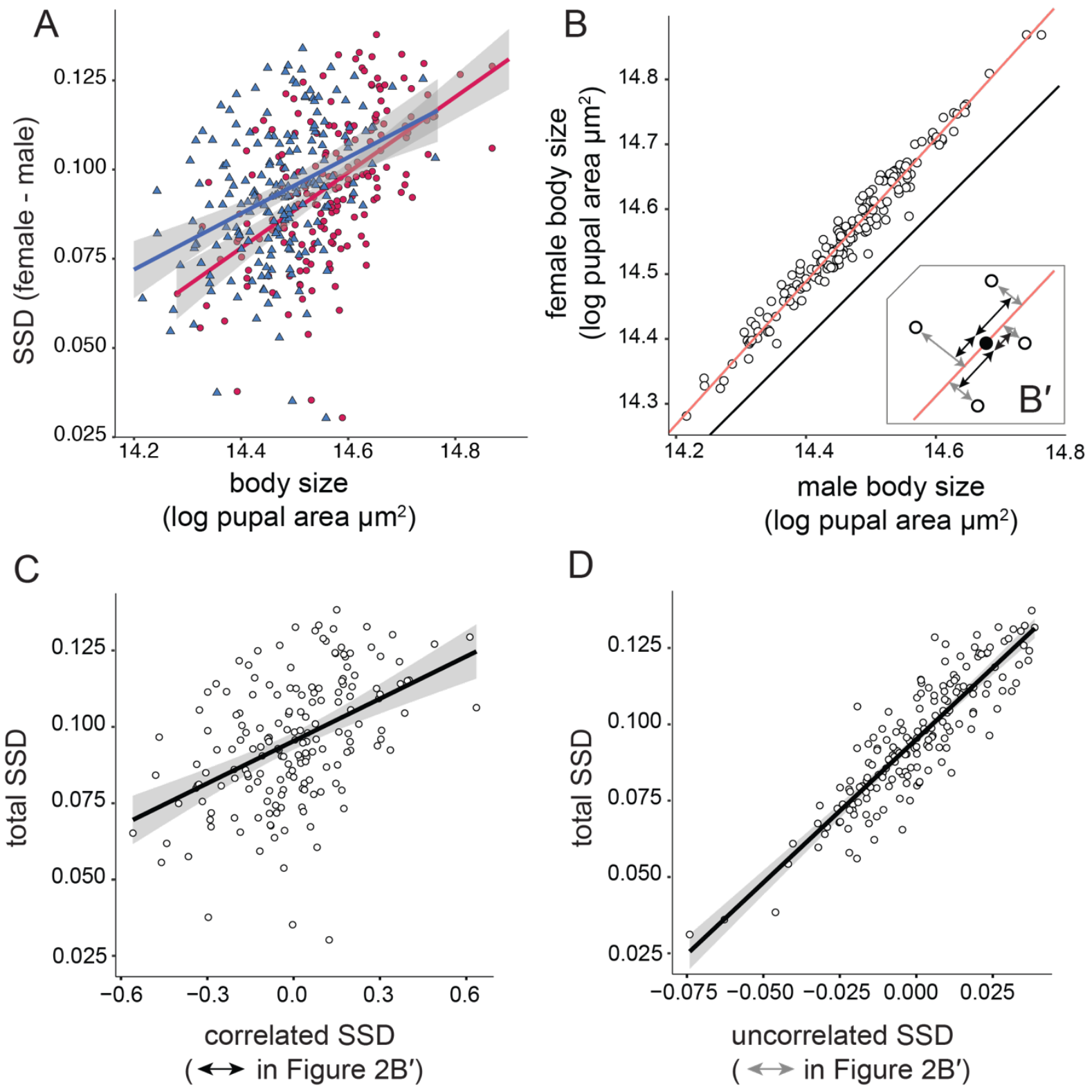

Figure 2: Patterns of SSD variation among lineages. (A) OLS linear regression between female (red circle) and male (blue triangle) size and SSD. Variation in SSD is due more to variation in female $\left(R^{2}=0.29\right.$, $95 \% \mathrm{Cl}: 0.18-0.40)$ than male $\left(R^{2}=0.29,95 \% \mathrm{Cl}: 0.18-0.40\right)$ size. (B) Major axis regression of female on male size (red line, slope $=1.1$ ). SSD increases as lineage pupal size increases, although more so in females than in males. Black line is $x=y$. ( $B^{\prime}:$ Inset) Variation in SSD can be explained by variation that is correlated with sex-averaged lineage size (correlated SSD, black arrows) and variation that is uncorrelated with sex-averaged lineage size (uncorrelated SSD, gray arrows). Solid point represents the bivariate mean female and male body size among all lineages. (C \& D) OLS linear regression of total SSD against correlated SSD (C) and uncorrelated SSD (D). Correlated SSD accounts for $22 \%$ variation in variation in SSD among lineages $\left(R^{2}=0.22\right)$ whereas uncorrelated SSD accounts for $78 \%$ variation in variation in SSD among lineages $\left(R^{2}=0.78\right)$. Gray shading is $95 \%$ confidence interval of the fit. 


\section{SSD increases with sex-averaged body size among lineages}

Male and female size are tightly correlated among lineages (Pearson's correlation $R=0.98, p<2.2 e-$

16, Fig. 2B). If variation in SSD due is more to variation in female size than male size, it follows that regressing female size ( $y$-axis) against male size ( $x$-axis) should yield a slope significantly greater than 1. Major Axis (MA) regression between female and male body size supported this prediction (slope= 1.10, $95 \% \mathrm{Cl}=1.07-1.13$; Fig. 2B). The observation that females lie above the line $y=x$ (Fig. 2B) indicates that females are larger than males among the lineages. The observation that the slope of this line is $>1$ indicates that SSD increases with sex-averaged body size among lineages (OLS, $P=$ 8.062e-09, Fig. S1) due to a disproportionate increase in female size. (Here, sex-averaged body size refers to body size independent of sex, that is $(\bar{m}+\bar{f}) / 2$, where $\bar{m}$ and $\bar{f}$ are predicted male and female body size respectively). Importantly, this suggests that there are two factors that generate variation in SSD among lineages: Variation in SSD that is correlated with variation in sex-averaged body size among lineages, and variation in SSD that is uncorrelated with variation in sex-averaged body size. We refer to these effects as correlated-SSD and uncorrelated-SSD, respectively. In Figure $\mathbf{2} \mathbf{B}^{\prime}$, correlated-SSD is the variation in SSD generated as lineages move up or down the red line (black arrows), while uncorrelated-SSD is the variation in SSD generated by lineage lying above or below the red line (grey arrows).

To calculate the extent to which variation in SSD is due to effects that are correlated and uncorrelated with sex-averaged body size, we first extracted the fitted values and residuals for each lineage from the MA regression of female on male body size. The $R^{2}$ of the linear relationship between the fitted values and SSD captures the proportion of SSD variation that is due to variation in sex-averaged body size (correlated-SSD), while the $R^{2}$ of the linear relationship between the residuals and SSD captures the proportion of SSD variation that is independent of variation in sexaveraged body size (uncorrelated-SSD). Because total variation in SSD is a combination of both variation that is correlated and uncorrelated with variation in sex-averaged body size among lineages, the adjusted $R^{2}$ of these two relationships sum to one. We found that the $R^{2}$ for the sex- 
averaged dependent effect was 0.1832 (95\% Cl: 0.12-0.32) (Fig. 2C), while the $R^{2}$ for the sexaveraged independent effect was 0.8107 (95\% Cl: 0.73-0.84) (Fig. 2D). Consequently, while variation in sex-averaged body size among lineages partially explains variation in SSD, variation due to difference in male or female size independent of sex-averaged body size explains variation in SSD to a greater extent.

\section{Females have greater nutritionally-induced size plasticity than males}

We first explored the factors that generate variation in SSD that are correlated with variation in sex-averaged size among lineages (correlated-SSD). Because female size is more variable among lineages than is male size, it follows that females are more sensitive to factors that influence size than are males. A key environmental determinant of body size in all animals is nutrition during growth and development. We therefore tested whether females are more sensitive than males to variation in developmental nutrition, using the difference in average male or female size between fed and starved treatments as a measure of the male or female plasticity for each lineage. In nearly all of the DGRP lineages, females had higher nutritionally-induced size plasticity than males (Fig. 3A \& B), and there was significant interaction between the effects of sex and starvation treatment on body size across lineages (Table 2, Model 3: $P_{\text {sex }}{ }^{*}$ starvation treatment $<0.0001$ ), indicating sex-specific plasticity. If females are generally more sensitive to size-regulatory factors than males, whether those factors are environmental or genetic, then this increased sensitivity may account for variation in SSD that is correlated with the sex-averaged body size of a lineage. 

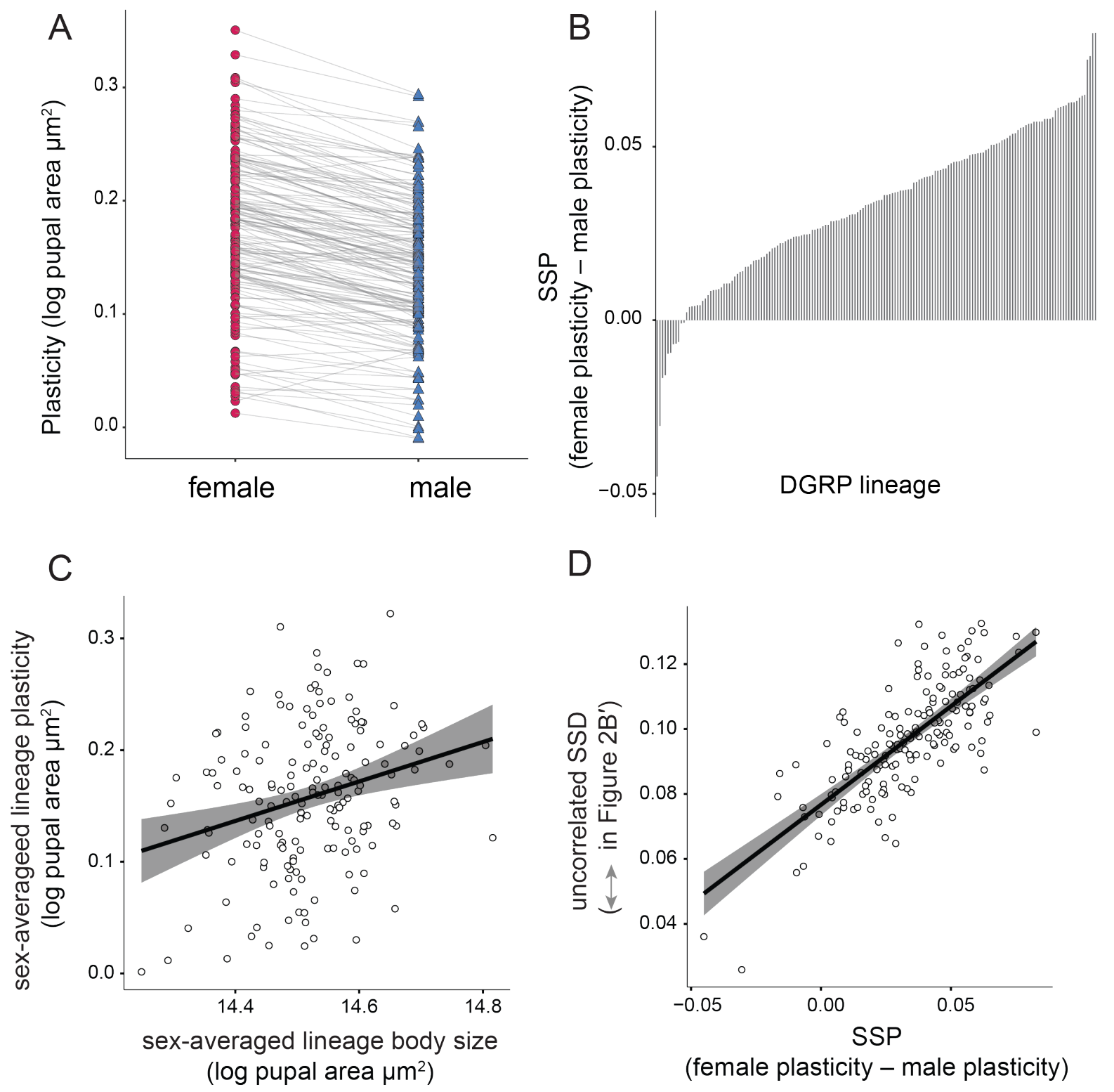

Figure 3. Patterns of SSP variation among lineages. (A) Female and male size plasticity for each of the 186 lineages. Estimates of plasticity use Best Linear Unbiased Predictions (BLUPs) of body size from a mixed model linear regression on fed and starved flies (Table 1: Model 1).. (B) Ranking plot of SSP for these lineages, where SSP is female plasticity - male plasticity (C) OLS linear regression of sex-averaged lineage body size against sex-averaged lineage plasticity. The relationship is significant but lose $\left(R^{2}=0.07\right.$, $P=0.0005)$. (D) OLS linear regression between uncorrelated SSD and SPP among lineages. The relationship is strong and highly significant. $\left(R^{2}=0.56, P<2.2 \mathrm{e}-16\right)$. Gray shading is $95 \%$ confidence interval of the fit. 


\section{Sex Specific Plasticity covaries with Sexual Size Dimorphism independent of lineage body size}

One simple hypothesis as to why females are more plastic than males is that females are larger than males and larger individuals are more plastic than smaller individuals. Indeed, there is a significant relationship between sex-averaged size and sex-averaged size plasticity among lineages

(Fig 3C). However, while significant, this relationship is weak $\left(R^{2}=0.07, P=0.0005\right)$, suggesting that SSP is not simply a reflection of the fact that females are bigger than males. To address this further, we explored how variation in SSP covaries with SSD independently of sex-averaged size, i.e. uncorrelated SSD (Fig. 2D). We first tested whether SSP varied significantly among lineages, and found that it did: A linear mixed effect model that included random variation in SSP (sex and starvation interaction) among lineages was a significantly better fit to the data than one that did not (Table 2). (Best Linear Unbiased Predictions: fed male body size $=14.47$, fed female body size $=$ 14.56 , starved male body size $=14.42$, starved female body size $=14.39$ ).

We then whether variation in SSP among lineages correlates with the (1) overall variation in SSD among lineages, and (2) variation in SSD independent of sex-averaged size (uncorrelated-SSD). In as much as variation in SSD among isogenic lineages is due to genotype, there was a positive genetic correlation between SSD and SSP, consistent with the condition dependent hypothesis of SSD (Fig .3D; Pearson's $R=0.75$ ). Further, an OLS linear regression of SSP against uncorrelated-SSD was significant with an adjusted $R^{2}$ of 0.56 . Thus variation in SSP among lineages explains the majority of variation in SSD that is independent of sex-averaged lineage pupal size.

\section{Discussion}

Sexual size dimorphism is widespread in animals. Among the possible evolutionary mechanisms that generate sex-specific size differences, the condition dependence hypothesis proposes that when the larger sex experiences stronger directional selection on body size, that sex will have greater fitness benefits if it can exploit environmental conditions favourable to growth $[14,16,17]$, 
resulting in sex-specific size plasticity $[12,19,20,25]$. Numerous comparative studies demonstrate a correlation between SSD and sex-specific size plasticity among traits within species [12], among closely related species $[18,20,26]$, and among populations within species $[8,27]$. Perhaps because of the correlative nature of these data, the condition dependence hypothesis is often discussed as a process that shapes SSD rather than one that generates it in the first place. Sex specific plasticity, however, de facto generates SSD, and so the condition dependent hypothesis can explain the evolution of SSD if selection for SSD targets the same developmental-genetic mechanisms that generate SSP. This predicts a positive genetic correlation between variation for SSD and SSP among individuals within a population. This is problematic to test, however, because SSD is a populationlevel, not an individual-level, measure. To circumvent this complication, we estimated SSD, SSP, and their covariation from populations of isogenic Drosophila lineages, where body size variation within lineages was induced by manipulating access to food. We found significant variation in femalebiased SSD among the lineages, and a tight positive correlation between SSD and female-biased SSP. That is, in genotypes where females are larger relative to males and SSD is more pronounced, independent of the sex-averaged body size of the lineage, females are correspondingly more plastic. The genetic covariation between SSD and SSP is consistent with the hypothesis that both are regulated by the same developmental mechanisms, and this pleiotropy has important implications for origin and evolution of SSD, which we explore below.

\section{Proximate mechanisms for Sex Specific Plasticity and Sexual Size Dimorphism}

Previous studies have used a variety of data to test the condition dependent hypothesis, correlating SSD with SSP among multiple traits within species $[12,28]$, or correlating SSD with SSP for a single trait among populations $[8,27]$ or species $[20,21]$. However, while these correlational studies provide general support for the condition dependence hypothesis, they cannot determine if condition dependence of sexually-dimorphic traits arise as a consequence of the dimorphism, or if condition dependence generates sexual dimorphism in plastic traits [16]. In order to distinguish between these two hypotheses it is necessary to have some understanding of the developmental 
genetic mechanisms that regulate both SSD and SSP. Our study goes some way to addressing this, by demonstrating a tight genetic correlation between SSD and SSP. There are two major causes of genetic correlation: linkage disequilibrium (LD) or pleiotropy. The DGRP population of $D$. melanogaster in our study has a rapid decay of LD, with an average squared correlation below 0.2 at just 10 base pairs on the autosomes [29]. Consequently, it seems unlikely that the genetic correlation we observe between SSD and SSP among lineages is the result of linkage.

A number of recent studies support the hypothesis that SSD in Drosophila is generated by the same developmental-genetic mechanisms that regulate nutritional plasticity of body size; specifically the insulin/IGF-signalling (IIS) and TOR-signalling pathways (see [30-32]. The IIS responds to circulating insulin-like peptides (dILPS in Drosophila), which are released in a nutrient-dependent manner and bind to the insulin receptors (InR) of dividing cells [33]. Binding activates a signaltransduction pathway that ultimately controls the expression of genes involved in regulating cell survival, growth, and proliferation [34]. The TOR-signalling pathway has a similar effect, but is regulated more directly by circulating amino acids $[32,35]$. There is considerable cross-talk between the two pathways so that they work together to tune organismal growth and development in response to the nutritional environment [36].

In Drosophila, SSD is eliminated in flies carrying a hypomorphic mutation of $\operatorname{InR}$ [37], which indicates that a functional IIS-pathway is necessary to generate the difference in body size between males and females. Further, fat body-specific knock-down of the sex-determining gene Transformer - which produces protein in females but not in males - results in the retention of dILP2 in the insulin-producing cells in the brains of females but not males [38]. Retention of dILP2 correlates with lower circulating levels of dILP2, and these females have a correspondingly reduced body size. These data suggest that females are larger than males because they have higher levels of circulating dILPs when well fed. Subsequent work has demonstrated that females, but not males, increase body size when reared on a very-high protein diet, and that this elevated sex-specific plasticity is also 
dependent on dILP2 and Transformer [39]. Collectively, these data support the hypothesis that both SSD and SSP are regulated by the IIS-pathway in Drosophila. An added nuance, however, is that the effect of nutrition on body size depends not just on food quantity but also food quality. Specifically, females, but not males, reduce body size when reared on high sugar diets ([40]although see [39]), and this appears to reflect sex-specific differences in the activity of the TOR-signalling pathway $[41,42]$.

Sexual dimorphism in the size of sexually-dimorphic traits in other systems also appears to be regulated by the IIS pathway. The horns of male rhinoceros beetle (Trypoxylus dichotomus) show heightened insulin-sensitivity and corresponding nutritional sensitivity relative to other traits in the body, with knock-down of $\operatorname{InR}$ having a greater effect on male horn size - reducing the sexual dimorphism [43]. Similarly, the size of the exaggerated mandibles of male broad-horned flour beetle (Gnatocerus cornutus) is regulated by a specific ILP (GcorILP2), which is expressed in a conditiondependent manner [44]. Thus, increased activity of the insulin-signaling pathway, either systemically or in specific tissues, appears to account for sex-specific condition-dependent increases in trait size among a diversity of insects.

\section{Evolutionary implications of pleiotropy for SSP and SSD}

The observation that sexual size dimorphism in Drosophila is generated by sex-specific plasticity has important implications for our understanding of the evolution of sexual dimorphism in general and SSD specifically. In particular, it suggests that selection targets mechanisms that conditionally increase trait size in one sex over the other. Why the conditional increase in trait size is favored only in one sex depends on the mode of selection. For sexually-dimorphic traits that are used by males to attract or compete for females and are subject to sexual selection, heightened conditionality of trait expression ensures that the trait is a reliable and sensitive indicator of male quality[43]. In flies and other insects, however, SSD appears to a consequence of fecundity selection. Female reproductive success is limited by the number of eggs she lays, and fecundity increases with body size [39,45-47]. 
Further, artificial selection on fecundity in female Drosophila leads to an increase in SSD [48]. The effect of male body size on fitness in Drosophila is much weaker or undetectable $[39,45]$, although this may be because male mating success is limited by access to females, and studies exploring the effect of male body size on fecundity used single pair matings $[39,45]$. While larger males may have slight reproductive advantage under scramble competition for matings [49], it is possible that males on high quality diets utilize mechanisms other than body size to enhance fitness, for example by reducing developmental time or increasing the number of matings. Regardless of the form or target of selection, however, selection favouring SSP in size will necessarily generate SSD, and our data suggest that selection targets the same mechanisms to result in both..

Our data showing a genetic correlation between SSD and SSP also has interesting implications for Rensch's rule, which states that male-biased SSD increases with overall body size, while femalebiased SSD decreases with overall body size, among species or populations [5]. The general explanation for this phenomenon is that male body size varies more than female body size among populations and species, a pattern that has been hypothesized to result from greater plasticity in male size [50]. Interspecific variation in SSD consistent with Rensch's rule is common in vertebrates [51,52], but is also seen in several invertebrate groups, including the Drosophilidae [53]. Among Drosophila genotypes, however, we found female size to be more variable than male size, and so female-biased SSD increases with overall body size among lineages - the reverse of Rensch's rule (albeit applied to genotypes rather than populations or species). The observation that the generally female-biased interspecific variation in SSD among Drosophilidae follows Rensch's rule, but that intraspecific variation in SSD exhibits a reverse Rensch's rule, suggests that Rensch's rule cannot be explained by greater plasticity in males versus females. Indeed, there is a general trend in both vertebrates and invertebrates that the larger sex is more plastic than the smaller sex $[18,22]$. If Rench's rule were due to sex-specific plasticity, then we should observe Rensch's rule only when males are larger than females, but not when females are larger than males. 


\section{Conclusions}

We tested whether the condition dependence hypothesis explains sexual size dimorphism using individual variation in a population of Drosophila melanogaster. We found that SSD and SSP shows a strong positive correlation, suggesting shared developmental-genetic mechanisms. We further suggest that the selective pressures that generate the SSD act on the same developmental mechanisms that regulate nutritionally-induced body size plasticity. In other words, the observed sexual-size dimorphism may be a consequence of sex-specific selection favouring increased body size as condition improves in females but less so in males. Full testing of this hypothesis requires identification of the loci that underlie variation in both SSD and SSP among these Drosophila lineages. More generally, however, our study makes it clear that to fully understand that selective pressures that generate SSD, it is necessary to also understand that developmental-genetic mechanisms those selective pressures target.

\section{Methods}

\section{Fly stocks}

Flies come from the Drosophila Genetic Reference Panel (DGRP), consisting of more than 200 inbred Drosophila melanogaster lineages generated from a natural population in Raleigh, NC, USA, after 20 generation of full-sib mating [29]. Each lineage discussed in this paper refers to a specific isogenic line in the DGRP with a unique genotype.

\section{Starvation treatment}

For each DGRP lineage, flies were reared following previously publish protocols $[24,54,55]$. Eggs were collected over $12-20 \mathrm{~h}$ and transferred in lots of 50 into $7 \mathrm{ml}$ fly food, and the larvae were reared at $22^{\circ} \mathrm{C}$, in on standard cornmeal-molasses medium until the starvation treatment was applied. Larvae were removed from food at precisely timed developmental stages and starved for either $0-24 \mathrm{~h}$ or $24-48 \mathrm{~h}$ before pupation to generate variation in body size. Because larvae stop feeding approximately $24 \mathrm{~h}$ before pupation [56], larvae removed from the food $0-24 \mathrm{~h}$ before 
pupation were essentially allowed to feed ad libitum and are referred to as 'fed' flies. In contrast, larvae removed from the food 24-48h before pupation were starved during the period when adult body size is affected by nutrition [57], and are referred to as 'starved' flies. After being removed from the food, larvae were transferred to an empty vial containing a wet cotton plug and left until pupariation. Pupae were then transferred to individual $2.5 \mathrm{~mL}$ Eppendorf tubes until eclosion to allow us to associate each adult fly with its pupal case. After the flies emerged as adults from their pupal cases, they were sexed and the pupal case was imaged and size quantified using semiautomated software developed in the Shingleton lab [58]. We use area of the pupal silhouette (dorsal view) as a proxy for adult body size $[24,54,55]$. Flies were collected in nine temporal blocks, with one lineage repeated across all blocks to serve as a control.

\section{Statistical analyses}

All analyses were conducted in R (v. 4.0.3), and the script and data used in the analyses provided on Dryad. Prior to analysis, data were filtered such that each sex-treatment-lineage combination had at least 10 pupal area measurements. After filtering, the remaining DGRP lineages were analyzed. All measurements were log transformed prior to analysis.

We used a Mixed Linear Model (R package: Ime4) [59] to test for genetic variation in Sexual Size Dimorphism (SSD) and Sex Specific Plasticity (SSP).

For SSD we used only the data from the fed flies and fit the models:

$$
\begin{gathered}
\text { Model 1: } P=S+(1 \mid B)+(1+S \mid L)+\varepsilon \\
\text { Model 2: } P=S+(1 \mid B)+(1 \mid L)+\varepsilon
\end{gathered}
$$

where $P$ is body size, $S$ is sex, $B$ is block (random factor), $L$ is lineage (random factor), and $\varepsilon$ is error. The two models differ only in whether there is random variation in the effect of sex on body size among lineages (Model 1), or not (Model 2); that is whether SSD varies among lineages. We compared the models using a log likelihood ratio test and parametric bootstrapping. 
For SSP, we used the data from the fed and starved flies and fit the models:

$$
\begin{aligned}
& \text { Model 3: } P=S \cdot D+(1 \mid B)+(1+S \cdot D \mid L)+\varepsilon \\
& \text { Model 4: } P=S \cdot D+(1 \mid B)+(1+\mathrm{S}+D \mid L)+\varepsilon
\end{aligned}
$$

where $D$ is diet (fed or starved). The two models differ in whether there is random variation in the interaction between the effects of sex and diet on pupal size among lineages (Model 1), or not (Model 2); that is whether SSP varies among lineages. Again, we compared the models using a log likelihood ratio test and parametric bootstrapping.

For the analysis of genetic variation of SSD, we used the Best Linear Unbiased Prediction (BLUP) values for each lineage and sex from Table 1: Model 1 to estimate predicted male and female size ( $\widehat{P}_{\text {male }}$ and $\widehat{P}_{\text {female }}$ respectively) for each lineage for both fed and starved flies separately.

Predicted sex-adjusted body size for fed flies in each lineage was calculated as:

$$
\hat{P}_{\text {lineage }}=\hat{P}_{\text {female }}+\hat{P}_{\text {male }} / 2
$$

Sexual size dimorphism for a lineage was calculated as:

$$
S S D=\hat{P}_{\text {female.fed }}-\hat{P}_{\text {male.fed }}
$$

Nutritionally-induced size plasticity for each sex within a lineage was calculated as:

$$
\begin{gathered}
\Delta_{\text {female }}=\hat{P}_{\text {female.fed }}-\hat{P}_{\text {female.starved }} \\
\Delta_{\text {male }}=\hat{P}_{\text {male.fed }}-\hat{P}_{\text {male.starved }}
\end{gathered}
$$

Mean sex-adjusted size plasticity for each lineage was calculated as:

$$
\Delta_{\text {lineage }}=\Delta_{\text {female }}+\Delta_{\text {male }} / 2
$$

Sex-specific size plasticity for a lineage was calculated as: 


$$
S S P=\Delta_{\text {female }}-\Delta_{\text {male }}
$$

These values were then used to examine the relationship between female and male size using Major Axis (MA) regression (R package: smartr) [60], and the fitted and residual values for this relationship were used to analyze sex-averaged size dependent and sex-averaged size independent measures of SSD (referred to as correlated and uncorrelated SSD, respectively). We then used ordinary least squares (OLS) regression to explore the relationship between total SSD and uncorrelated and correlated SSD, lineage mean size and lineage mean plasticity, and between SSP and correlated and uncorrelated SSD.

\section{Acknowledgements}

We would like to thank all the members of the Shingleton and Frankino labs for their assistance and support during the collection of these data. Particular thanks to Omid Ziabari, Sonya Messer and Chris Edomwande for organizing the collection of the pupal measurements.

\section{Funding}

This research was funded by NSF award IOS-1901727 and IOS-1952385 to AWS and NSF award IOS-1558098 and 2019 GEAR Award from the University of Houston to WAF. 
bioRxiv preprint doi: https://doi.org/10.1101/2021.06.16.448738; this version posted June 17,2021. The copyright holder for this preprint (which was not certified by peer review) is the author/funder, who has granted bioRxiv a license to display the preprint in perpetuity. It is made available under aCC-BY-NC 4.0 International license.

\section{Tables}

Table 1: Effect of including random variation in sex-by-lineage interaction on the fit of the relationship between body size and sex.

\begin{tabular}{|c|l|c|c|l|c|}
\hline \multicolumn{1}{|c|}{ Model $^{\mathbf{a}}$} & DF $^{\mathbf{b}}$ & AIC $^{\mathbf{c}}$ & BIC $^{\mathbf{c}}$ & Likelihood Ratio $^{\mathbf{c}}$ & $\boldsymbol{P}^{\mathbf{d}}$ \\
\hline Model 1: $P=S+B+S \bullet L$ & 7 & -11764 & -11715 & 40.2 & $<0.001$ \\
\hline Model 2: $\mathrm{P}=\mathrm{S}+\mathrm{B}+\mathrm{L}$ & 5 & -11728 & -11693 & & \\
\hline
\end{tabular}

${ }^{a} \mathrm{P}$ is the log transformed pupal case area, $\mathrm{S}$ is the sex, $\mathrm{B}$ is block (random effect), $\mathrm{L}$ is the lineage (random effect). The models differ by having random sex-by-lineage effects (model 1 ) versus random lineage effects (model 2).

${ }^{b}$ Estimated degrees of freedom for each model.

${ }^{\mathrm{C}} \mathrm{AIC}, \mathrm{BIC}$, and likelihood ratio calculated using ML fit.

${ }^{\mathrm{d}} \mathrm{P}$-value calculated by parametric bootstrapping using $\mathrm{ML}$ fit.

Table 2: Effect of including random variation in sex-by-starvation-by-lineage interaction on the fit of the relationship between body size, sex, and starvation treatment.

\begin{tabular}{|c|l|c|c|l|c|}
\hline \multicolumn{1}{|c|}{ Model $^{\mathbf{a}}$} & $\mathbf{D F}^{\mathbf{b}}$ & AIC $^{\mathbf{c}}$ & BIC $^{\mathbf{c}}$ & Likelihood Ratio $^{\mathbf{c}}$ & $\boldsymbol{P}^{\mathbf{d}}$ \\
\hline Model 3: $P=S \bullet D+B+S \bullet D \bullet L$ & 16 & -19252 & -19137 & 15.11 & 0.004 \\
\cline { 1 - 5 } Model 4: $P=S \bullet D+B+(S+D) \bullet L$ & 12 & -19259 & -19160 & & \\
\hline
\end{tabular}

${ }^{a} P$ is the log transformed pupal case area, $S$ is the sex, $D$ is the number of days of starvation, $B$ is the collecting block (random effect), $L$ is the lineage (random effect). The models differ by having random variation in SSP among lineages (sex-by-starvation-by-lineage interaction, model 3) versus random additive effect of sex and starvation among lineages.

${ }^{\mathrm{b}}$ Estimated degrees of freedom for each model.

${ }^{\mathrm{C}} \mathrm{AIC}, \mathrm{BIC}$, and likelihood ratio calculated using ML fit.

${ }^{\mathrm{d}} \mathrm{P}$-value calculated by parametric bootstrapping using $\mathrm{ML}$ fit. 


\section{References}

1. Blanckenhorn WU, Stillwell RC, Young KA, Fox CW, Ashton KG. 2006 When Rensch meets Bergmann: Does sexual size dimorphism change systematically with latitude? Evolution 60, 20042011. (doi:10.1554/06-110.1)

2. Fairbairn DJ. 2016 Sexual Dimorphism. Sex Recomb Mating Syst Article Titles S , 105113. (doi:10.1016/b978-0-12-800049-6.00157-8)

3. Lindenfors P, Gittleman JL, Jones KE. 2007 Sexual size dimorphism in mammals. In Sex, Size and Gender Roles: Evolutionary Studies of Sexual Size Dimorphism (eds DJ Fairbairn, WU Blanckenhorn, T Székely), Oxford, UK: Oxford University Press.

4. Székely T, Lislevand T, Figuerola J. 2007 Sexual size dimorphism in birds. In Sex, Size and Gender Roles: Evolutionary Studies of Sexual Size Dimorphism (eds DJ Fairbairn, WU Blanckenhorn, T Székely), Oxford, UK: Oxford University Press.

5. Blanckenhorn WU, Meier R, Teder T. 2007 Rensch's rule in insects: patterns among and within species. In Sex, Size and Gender Roles: Evolutionary Studies of Sexual Size Dimorphism (eds DJ Fairbairn, WU Blanckenhorn, T Székely), Oxford, UK: Oxford University Press.

6. Rohner PT, Teder T, ESPERK T, Lüpold S, Blanckenhorn WU. 2017 The evolution of male-biased sexual size dimorphism is associated with increased body size plasticity in males. Funct Ecol 32, 581 591. (doi:10.1111/1365-2435.13004)

7. Butler MA, Schoener TW, Losos JB. 2000 The relationship between sexual size dimorphism and habitat use in Greater Antillean Anolis lizards. Evolution 54, 259-272. (doi:10.1111/j.00143820.2000.tb00026.x)

8. Cox RM, Calsbeek R. 2010 Sex-specific selection and intraspecific variation in sexual size dimorphism. Evolution 64, 798-809. (doi:10.1111/j.1558-5646.2009.00851.x)

9. Pearson D, Shine R, Williams A. 2002 Geographic variation in sexual size dimorphism within a single snake species (Morelia spilota, Pythonidae). Oecologia 131, 418 426. (doi:10.1007/s00442002-0917-5)

10. Andersson M. 1994 Sexual Selection. Princeton, NJ: Princeton University Press.

11. Andersson M. 1986 Evolution of condition-dependent sex ornaments and mating preferences: sexual selection based on viability differences. Evolution 40, 804816.

12. Bonduriansky R. 2007 The evolution of condition-dependent sexual dimorphism. Am Nat 169, 9 19. (doi:10.1086/510214)

13. Nur N, Hasson O. 1984 Phenotypic plasticity and the handicap principle. J Theor Biol 110, 275 297. (doi:10.1016/s0022-5193(84)80059-4)

14. Rowe L, Houle D. 1996 The lek paradox and the capture of genetic variance by condition dependent traits. Proc Royal Soc Lond Ser B Biological Sci 263, 1415-1421. (doi:10.1098/rspb.1996.0207)

15. Grafen A. 1990 Sexual selection unhandicapped by the fisher process. $J$ Theor Biol 144, 473-516. (doi:10.1016/s0022-5193(05)80087-6) 
bioRxiv preprint doi: https:/doi.org/10.1101/2021.06.16.448738; this version posted June 17, 2021. The copyright holder for this preprint (which was not certified by peer review) is the author/funder, who has granted bioRxiv a license to display the preprint in perpetuity. It is made available under aCC-BY-NC 4.0 International license.

16. Johnstone RA, Rands SA, Evans MR. 2009 Sexual selection and condition-dependence. $J$ Evolution Biol 22, 2387 2394. (doi:10.1111/j.1420-9101.2009.01822.x)

17. Bonduriansky R, Day T. 2003 The evolution of static allometry in sexually selected traits. Evolution 57, 2450 2458. (doi:10.1554/03-213)

18. Stillwell RC, Blanckenhorn WU, Teder T, Davidowitz G, Fox CW. 2010 Sex differences in phenotypic plasticity affect variation in sexual size dimorphism in insects: From physiology to evolution. Annu Rev Entomol 55, 227-245. (doi:10.1146/annurev-ento-112408-085500)

19. Bonduriansky R, Rowe L. 2005 Sexual selection, genetic architecture, and the condition dependence of body shape in the sexually dimorphic fly Prochyliza xanthostoma (Piophilidae). Evolution 59, 138-151. (doi:10.1111/j.0014-3820.2005.tb00901.x)

20. Rohner PT, Blanckenhorn WU. 2018 A Comparative Study of the Role of Sex-Specific Condition Dependence in the Evolution of Sexually Dimorphic Traits. Am Nat 192, E202-E215. (doi:10.1086/700096)

21. Teder T, TAMMARU T. 2005 Sexual size dimorphism within species increases with body size in insects. Oikos 108, 321 334. (doi:10.1111/j.0030-1299.2005.13609.x)

22. Badyaev AV. 2002 Growing apart: an ontogenetic perspective on the evolution of sexual size dimorphism. Trends Ecol Evol 17, 369-378. (doi:10.1016/s0169-5347(02)02569-7)

23. Sheldon BC, Merilä J, Lindgren G, Ellegren H. 1998 Gender and environmental sensitivity in nestling collared flycatchers. Ecology 79, 1939-1948. (doi:10.1890/0012-

9658(1998)079[1939:gaesin]2.0.co;2)

24. Stillwell RC, Dworkin I, Shingleton AW, Frankino WA. 2011 Experimental manipulation of body size to estimate morphological scaling relationships in Drosophila. J Vis Exp Jove, e3162 e3162. (doi:10.3791/3162)

25. Bonduriansky R. 2007 The genetic architecture of sexual dimorphism: the potential roles of genomic imprinting and condition-dependence. In Sex, Size and Gender Roles: Evolutionary Studies of Sexual Size Dimorphism (eds D Fairbairn, WU Blanckenhorn, T Székely), Oxford, UK: Oxford University Press.

26. Teder T, TAMMARU T, ESPERK T. 2008 Dependence of Phenotypic Variance in Body Size on Environmental Quality. Am Nat 172, 223 232. (doi:10.1086/589896)

27. Bonneaud C, Marnocha E, Herrel A, Vanhooydonck B, Irschick DJ, Smith TB. 2016 Developmental plasticity affects sexual size dimorphism in an anole lizard. Funct Ecol 30, 235-243. (doi:10.1111/1365-2435.12468)

28. Cotton S, Fowler K, Pomiankowski A. 2004 Condition dependence of sexual ornament size and variation in the stalk-eyed fly Cyrtodiopsis dalmanni (Diptera: Diopsidae). Evolution 58, 10381046. (doi:10.1554/03-514)

29. Mackay TFC et al. 2012 The Drosophila melanogaster Genetic Reference Panel. Nature 482, 173 178. (doi:10.1038/nature10811)

30. Vea IM, Shingleton AW. 2020 Network-regulated organ allometry: The developmental regulation of morphological scaling. Wiley Interdiscip Rev Dev Biology, e391. (doi:10.1002/wdev.391) 
bioRxiv preprint doi: https://doi.org/10.1101/2021.06 16.448738; this version posted June 17 2021. The copyright holder for this preprint (which was not certified by peer review) is the author/funder, who has granted bioRxiv a license to display the preprint in perpetuity. It is made available under aCC-BY-NC 4.0 International license.

31. Goberdhan DCI, Wilson C. 2003 The functions of insulin signaling: size isn't everything, even in Drosophila. Differentiation 71, 375-397. (doi:10.1046/j.1432-0436.2003.7107001.x)

32. Wullschleger S, Loewith R, Hall MN. 2006 TOR signaling in growth and metabolism. Cell 124, 471 484. (doi:10.1016/j.cell.2006.01.016)

33. Semaniuk U, Piskovatska V, Strilbytska O, Strutynska T, Burdyliuk N, Vaiserman A, Bubalo V, Storey KB, Lushchak O. 2020 Drosophila insulin-like peptides: from expression to functions - a review. Entomol Exp Appl (doi:10.1111/eea.12981)

34. Geminard C et al. 2006 Control of metabolism and growth through insulin-like peptides in Drosophila. Diabetes 55, S5 S8. (doi:10.2337/db06-s001)

35. Oldham S, Hafen E. 2003 Insulin/IGF and target of rapamycin signaling: a TOR de force in growth control. Trends Cell Biol 13, 79 85. (doi:10.1016/s0962-8924(02)00042-9)

36. Miron M, Lasko P, Sonenberg N. 2003 Signaling from Akt to FRAP/TOR Targets both 4E-BP and S6K in Drosophila melanogaster. Mol Cell Biol 23, 9117-9126. (doi:10.1128/mcb.23.24.91179126.2003)

37. Testa ND, Ghosh SM, Shingleton AW. 2013 Sex-specific weight loss mediates sexual size dimorphism in Drosophila melanogaster. Plos One 8, e58936. (doi:10.1371/journal.pone.0058936)

38. Rideout EJ, Narsaiya MS, Grewal SS. 2015 The Sex Determination Gene transformer Regulates Male-Female Differences in Drosophila Body Size. Plos Genet 11, e1005683.

(doi:10.1371/journal.pgen.1005683)

39. Millington JW, Brownrigg GP, Chao C, Sun Z, Basner-Collins PJ, Wat LW, Hudry B, MiguelAliaga I, Rideout EJ. 2021 Female-biased upregulation of insulin pathway activity mediates the sex difference in Drosophila body size plasticity. Elife 10, e58341. (doi:10.7554/elife.58341)

40. Shingleton AW, Masandika JR, Thorsen LS, Zhu Y, Mirth CK. 2017 The sex-specific effects of diet quality versus quantity on morphology in Drosophila melanogaster. Roy Soc Open Sci 4, 170375. (doi:10.1098/rsos.170375)

41. McDonald JMC, Nabili P, Thorsen L, Jeon S, Shingleton AW. 2021 Sex-specific plasticity and the nutritional geometry of insulin-signaling gene expression in Drosophila melanogaster. Evodevo 12, 6. (doi:10.1186/s13227-021-00175-0)

42. Millington JW, Wat LW, Sun Z, Basner-Collins PJ, Brownrigg GP, Rideout EJ. 2021 A low sugar diet enhances Drosophila body size in males and females via sex-specific mechanisms. Biorxiv, 2021.03.03.433819. (doi:10.1101/2021.03.03.433819)

43. Emlen DJ, Warren IA, Johns A, Dworkin I, Lavine LC. 2012 A mechanism of extreme growth and reliable signaling in sexually selected ornaments and weapons. Science 337, 860864. (doi:10.1126/science.1224286)

44. Okada Y, Katsuki M, Okamoto N, Fujioka H, Okada K. 2019 A specific type of insulin-like peptide regulates the conditional growth of a beetle weapon. Plos Biol 17, e3000541.

(doi:10.1371/journal.pbio.3000541) 
bioRxiv preprint doi: https:/doi.org/10.1101/2021.06.16.448738; this version posted June 17, 2021. The copyright holder for this preprint (which was not certified by peer review) is the author/funder, who has granted bioRxiv a license to display the preprint in perpetuity. It is made available under aCC-BY-NC 4.0 International license.

45. Lefranc A, Bundgaard J. 2000 The Influence of Male and Female Body Size on Copulation Duration and Fecundity in Drosophila Melanogaster. Hereditas 132, 243-247. (doi:10.1111/j.16015223.2000.00243.x)

46. Bergland AO, Genissel A, Nuzhdin SV, Tatar M. 2008 Quantitative trait loci affecting phenotypic plasticity and the allometric relationship of ovariole number and thorax length in Drosophila melanogaster. Genetics 180, 567 582. (doi:10.1534/genetics.108.088906)

47. Honěk A, Honek A. 1993 Intraspecific Variation in Body Size and Fecundity in Insects: A General Relationship. Oikos 66, 483. (doi:10.2307/3544943)

48. Reeve J, Fairbairn D. 1996 Sexual size dimorphism as a correlated response to selection on body size: An empirical test of the quantitative genetic model. Evolution 50, 19271938.

49. Partridge L, Hoffmann A, Jones JS. 1987 Male size and mating success in Drosophila melanogaster and D. pseudoobscura under field conditions. Anim Behav 35, 468476. (doi:10.1016/s0003-3472(87)80272-5)

50. Fairbairn D. 2005 Allometry for sexual size dimorphism: Testing two hypotheses for Rensch's rule in the water strider Aquarius remigis. The American Naturalist 166, S69 S84.

51. Abouheif E, Fairbairn DJ. 1997 A comparative analysis of allometry for sexual size dimorphism: Assessing Rensch's rule. Am Nat 149. (doi:10.1086/286004)

52. Fairbairn DJ. 1997 Allometry for Sexual Size Dimorphism: Pattern and Process in the Coevolution of Body Size in Males and Females on JSTOR. Annual Review Of Ecology And Systematics (doi:10.2307/2952508)

53. Blanckenhorn WU et al. 2007 Proximate causes of Rensch's rule: does sexual size dimorphism in arthropods result from sex differences in development time? Am Nat 169, 245257.

(doi:10.1086/510597)

54. Stillwell RC, Shingleton AW, Dworkin I, Frankino WA. 2016 Tipping the scales: Evolution of the allometric slope independent of average trait size. Evolution 70, 433 444. (doi:10.1111/evo.12865)

55. Myers EM, Frankino WA. 2012 Time in a Bottle: The Evolutionary Fate of Species Discrimination in Sibling Drosophila Species. Plos One 7, e31759.

(doi:10.1371/journal.pone.0031759)

56. Ghosh SM, Testa ND, Shingleton AW. 2013 Temperature-size rule is mediated by thermal plasticity of critical size in Drosophila melanogaster. Proc Royal Soc B Biological Sci 280, 20130174 20130174. (doi:10.1098/rspb.2013.0174)

57. Shingleton AW, Das J, Vinicius L, Stern DL. 2005 The temporal requirements for insulin signaling during development in Drosophila. Plos Biol 3, e289. (doi:10.1371/journal.pbio.0030289)

58. Shingleton AW, Estep CM, Driscoll MV, Dworkin I. 2009 Many ways to be small: different environmental regulators of size generate distinct scaling relationships in Drosophila melanogaster. Proc Royal Soc B Biological Sci 276, 2625-2633. (doi:10.1098/rspb.2008.1796)

59. Bates D, Mächler M, Bolker B, Walker S. 2014 Fitting linear mixed-effects models using lme4. Drug Alcohol Rev 36. (doi:10.1111/dar.12532) 
bioRxiv preprint doi: https://doi.org/10.1101/2021.06.16.448738; this version posted June 17, 2021. The copyright holder for this preprint (which was not certified by peer review) is the author/funder, who has granted bioRxiv a license to display the preprint in perpetuity. It is made available under aCC-BY-NC 4.0 International license.

60. Warton DI, Duursma RA, Falster DS, Taskinen S. 2012 smatr 3- an R package for estimation and inference about allometric lines. Methods Ecol Evol 3, 257 259. (doi:10.1111/j.2041210x.2011.00153.x) 


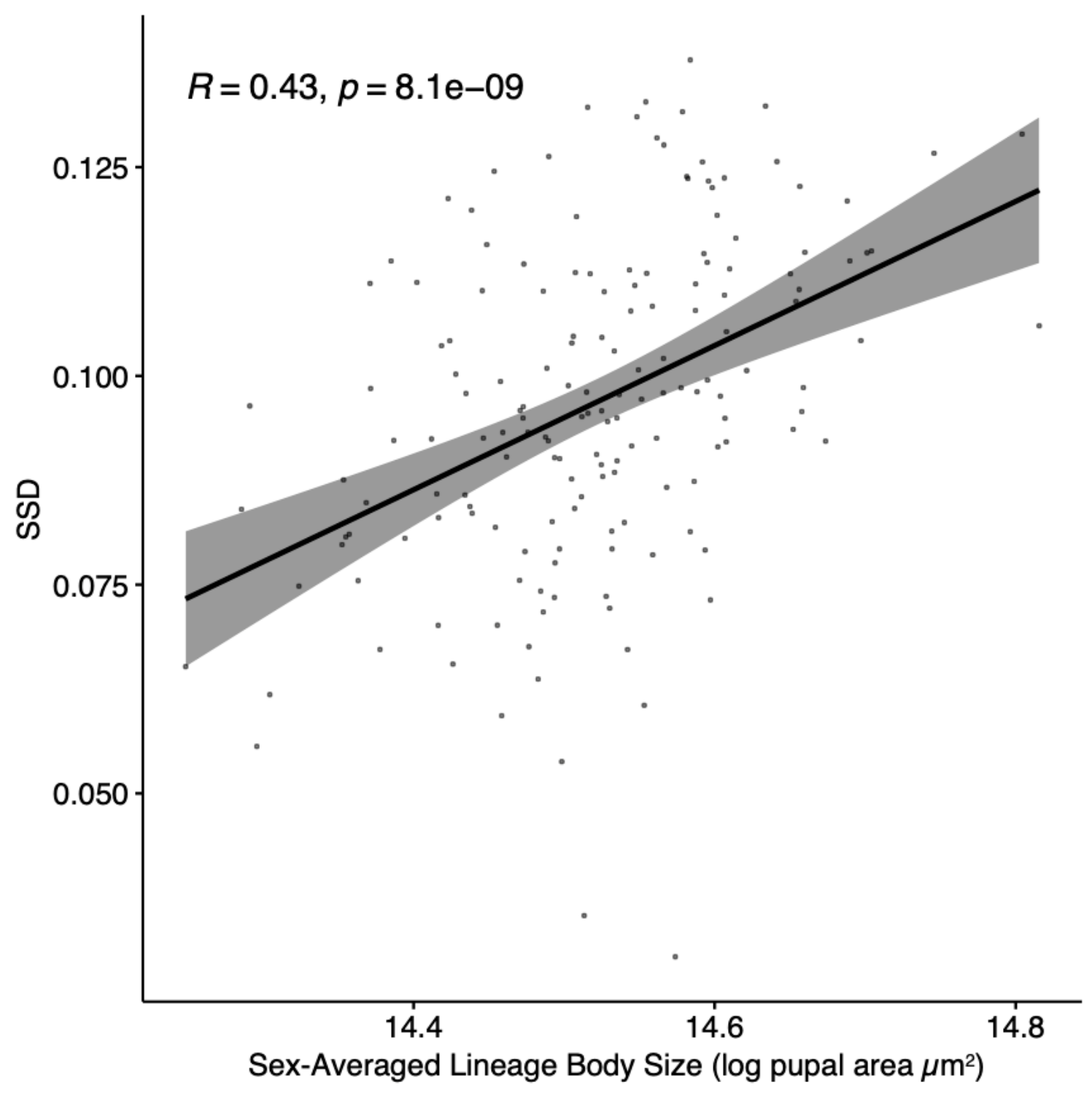

Supplementary Figure 1: The relationship between sex-averaged lineage body size and SSD. Line is an OLS linear regression. Shading is $95 \%$ confidence interval of the slope. 